\title{
Barriers to early mobilisation within Musculoskeletal and Colorectal Enhanced Recovery
}

\author{
Jones E.L, ${ }^{1}$ Wainwright T. $W^{2}$ Davies $S,{ }^{1}$ Porter $P_{,}^{3}$ Francis, N. $K^{4}$ \\ ${ }^{1}$ Physiotherapy, Yeovil District Hospital, Somerset BA21 4AT, UK; ${ }^{2}$ Orthopaedic Department, The Royal \\ Bournemouth Hospital, Bournemouth, BH7 7DW; ${ }^{3}$ Orthopaedic Surgery, Yeovil District Hospital, Somerset, BA21 \\ 4AT, United Kingdom; Colorectal Surgery, Yeovil District Hospital, Somerset, BA21 4AT, United Kingdom
}

\section{Introduction}

Enhanced Recovery After Surgery (ERAS) protocols emphasise the importance of early mobilisation after surgery. When applied in combination with pre operative and intra-operative ERAS elements early mobilisation is known to improve clinical and patient reported outcome. However, although early mobilisation is a well established element of ERAS its implementation can be hampered by clinical, organisational and emotive factors. The aim of this research is to survey staff opinion on the implementation process and challenges to early mobilisation across Musculoskeletal (MSK) and Colorectal (CR) wards at Yeovil Hospital.

\section{Results}

32 staff completed the survey; 17 MSK and 15 CR of a cohort of 40. CR staff reported unanimously that mobilising patients before breakfast could impact on length of stay while $53 \%$ of MSK staff believed that the timing of first mobilisation does not alter it, with $35 \%$ stipulating this should occur after breakfast. All staff felt competent and identified no training issues. The most common barriers identified were; staff availability ( $88 \%$ of MSK) and low blood pressure $(60 \%$ of CR and MSK). Other factors included nausea, pain and anaesthetic/analgesia type especially after knee surgery (figure 1). MSK staff reported that organisational multi-disciplinary changes should be implemented to overcome barriers (fig 2). MSK staff emphasise the role of the therapists to drive mobilisation while CR staff emphasised the role of nurses.

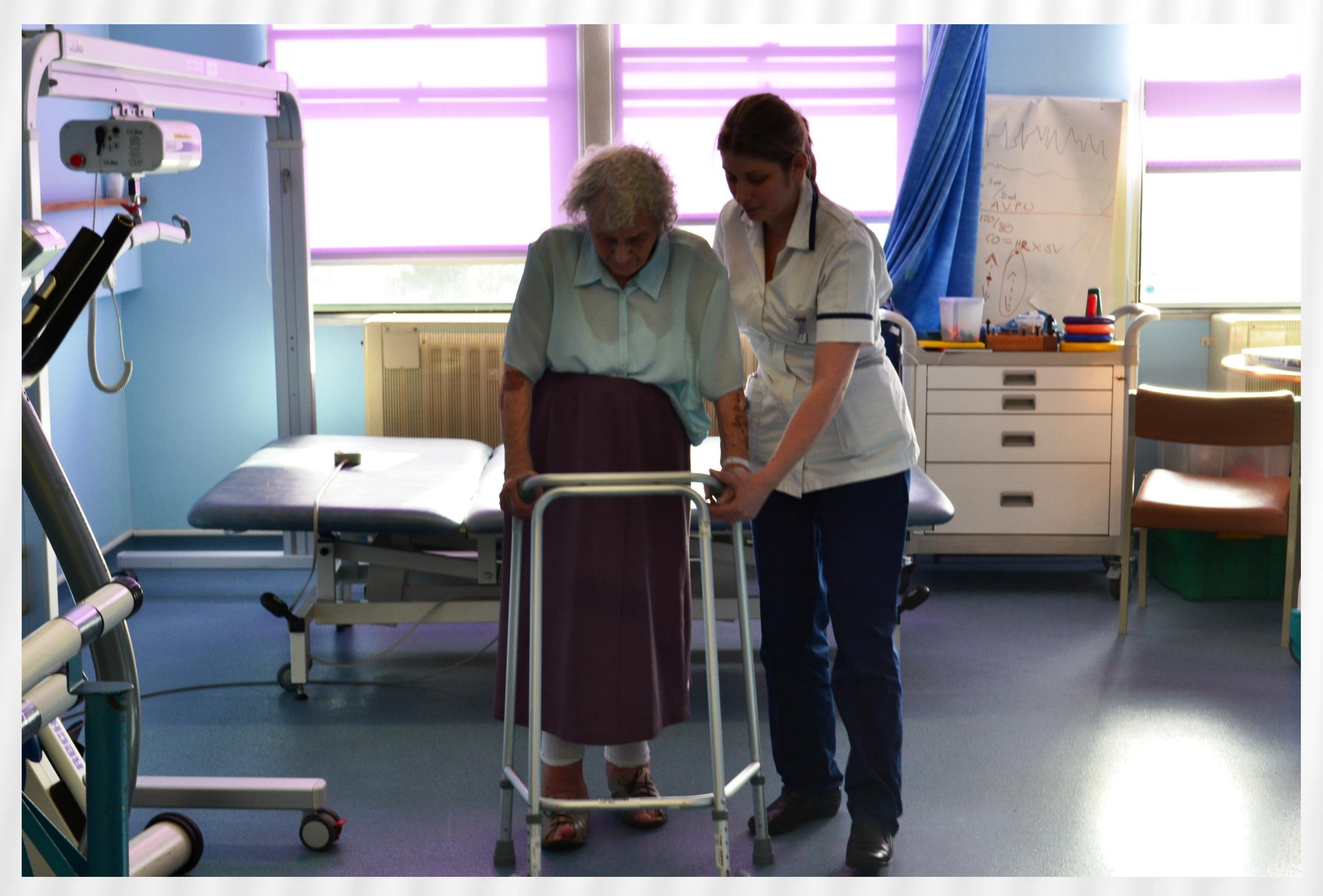

\section{Discussion}

Differences between MSK and CR staff opinion on the timing of mobilisation and barriers to mobilisation could be underpinned by: staffing patterns, beliefs, ERAS tradition, clinical reasoning and interdisciplinary working. This survey has demonstrated that greater knowledge exchange between specialities could be important. Further analysis is required to evaluate whether the timing of first mobilisation improves outcomes.

\section{Methods}

MSK and CR staff (nursing, health care assistant and therapy) were issued with paper surveys addressing: i) timing of mobilisation, ii) competency to mobilise day one post operative patients and iii) barriers in July 2013. Qualitative data was subjected top thematic analysis and shared and disparate views were considered across the two data sets. Themes were cross checked by SD.
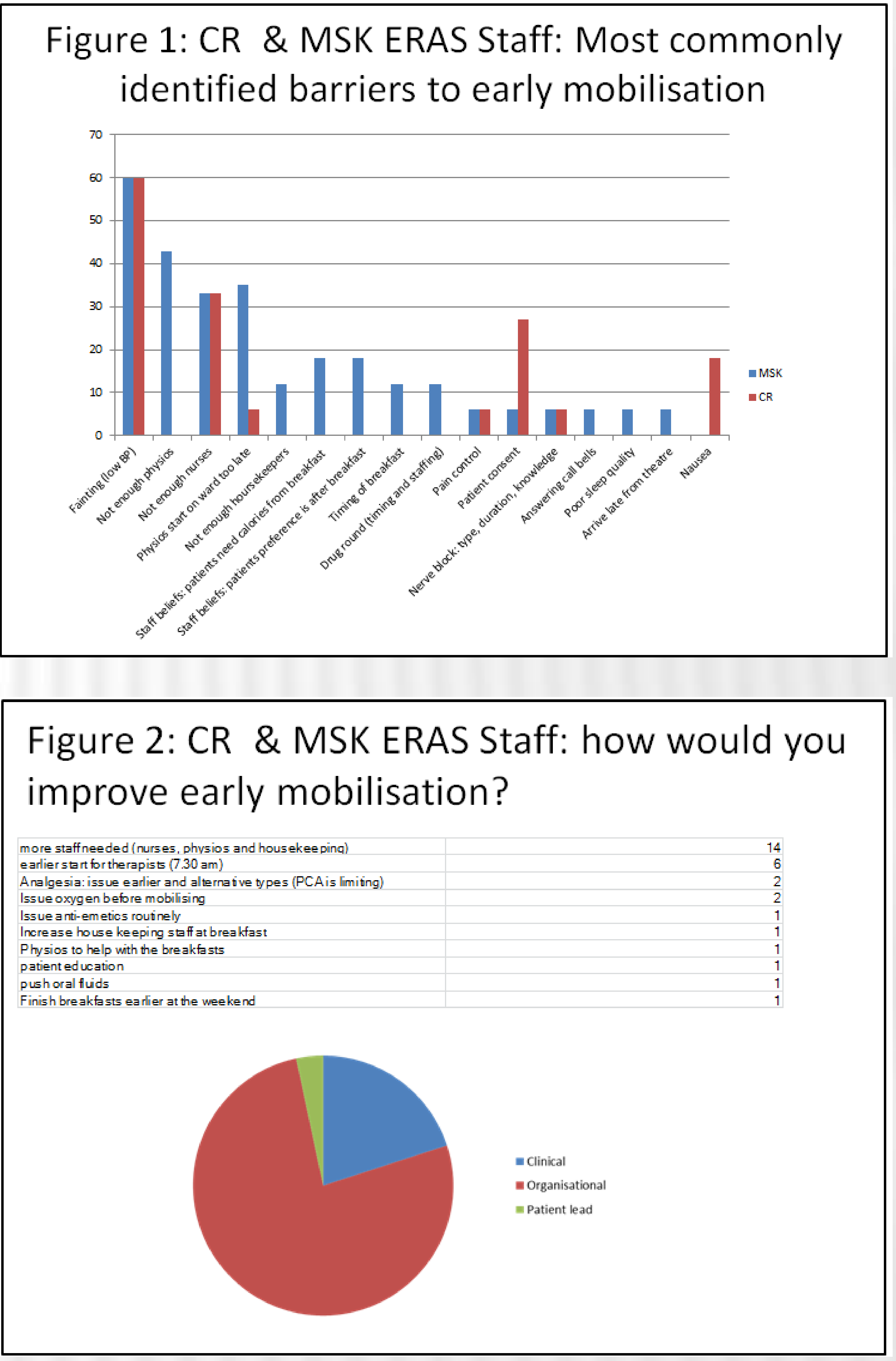

\section{Conclusion}

There is a difference in culture between Musculoskeletal and Colorectal staff in the timing of early mobilisation, and a divided opinion on whether this impacts LOS. Multiple challenges to early mobilisation can be overcome by a multi-disciplinary approach across specialities.

Contact: Nader.Francis@ydh.nhs.uk 